\title{
A Research on Cognitive Mechanism and Teaching Strategies of Mathematical Modeling
}

\author{
Huipeng Zhang ${ }^{1, a^{*}}$ \\ ${ }^{1}$ Department of mathematics and physics, Tianshi College, Tianjin New Technology Industrial Park \\ Development Zone Wuqing Road No.15source, 301700 \\ a2238552865@qq.com \\ *The corresponding author
}

Keywords: Mathematical modeling; Cognitive mechanism; Teaching strategies

\begin{abstract}
Mathematical modeling is the bridge using mathematics to solve practical problems. In recent years, colleges are actively exploring effective measures to deepen the reform of College Mathematics Education. At the same time, more attention is paid to cultivating college students' innovation ability of mathematical modeling. However, the cognitive mechanism of mathematical modeling and teaching strategy are still the problem not yet deeply studied, and study of this issue helps to enrich the mathematics learning psychology theory and develops theoretical mathematics problem solving theories in order to solve the problem of mathematical modeling so as to enhance the teaching effect and provide a theoretical basis and practical guidance. Thus, it has important theoretical significance and practical value.
\end{abstract}

\section{Introduction}

It is generally believed that the mathematical model is the mathematical simplification of the actual things, the abstract and simplified mathematical structure about some real world for a certain purpose, and the process of establishing the mathematical model is called mathematical modeling. Mathematical modeling is a practice of using mathematical methods to solve practical problems. It is to express the practical problems in mathematical ways, establish mathematical models and then use advanced mathematical methods and computer technology to solve them. Therefore, mathematical modeling is the process of describing the actual phenomenon in mathematical language. The National Mathematical Contest in Modeling (MCM) is jointly organized by the Higher Education Department of the Ministry of Education and the China Society for Industry and Applied Mathematics (CSIAM), held annually, It is one of the four major competitions for all colleges and universities in the country. The The purpose of the competition is to "encourage students to learn mathematics, improve the comprehensive ability for students to solve the practical problems by establishing mathematical models and the use of computer technology, to encourage students to actively participate in extracurricular scientific and technological activities, for developing knowledge and cultivating the spirit of creativity and cooperation, further promoting the reform of mathematics teaching system, teaching content and methods."

Cognition is the core concept of cognitive psychology and the process of information processing for a certain purpose in a certain psychological structure. Its essence is to study the psychological activities on the psychological mechanism of the physiological mechanism, to explore the various factors involved in the process of human information processing and the various stages of the experience and the cognitive factors of cognitive activity. The cognitive mechanism of mathematical modeling refers to the cognitive mechanism of the subject in the process of solving the practical problems by using the mathematical modeling method, including the general cognitive process of mathematical modeling, the cognitive differences in the mathematical modeling process and the mathematical modeling influencing factors and their ways of action and so on. The cognitive process of mathematical modeling has a very important research value. The cognitive mechanism of mathematical modeling and its teaching strategy are not yet studied deeply. It is urgent to carry out systematic research. Based on the problem of 
mathematical modeling, the cognitive mechanism of mathematical modeling and its teaching is proposed.

\section{Characteristics of Mathematical Modeling and Its Problem Representation}

Characteristics of Mathematical Modeling. The college students' mathematical modeling competition student-centered treating the practical problem as the main line, is a kind of college students' extracurricular scientific and technological activities aiming at cultivating students' innovation ability, and it has the following characteristics:

Openness and Initiative. The solution process, the solution tools and the results of the students' mathematical modeling contest are open. It focuses on cultivating students' innovative consciousness, innovative spirit and innovative thinking, thus breaking through the traditional teaching methods where teachers and teaching materials are the center, greatly mobilizing students' enthusiasm of study, and strengthening the students' practical ability.

Comprehensiveness and Applicability. College students' mathematical modeling activities are comprehensive and strong learning and training, which can effectively integrate the students' knowledge structure and further promote their initiative and enthusiasm for learning the follow-up courses. The titles of college students' mathematics modeling contest are coming from engineering technology and social economic life. It embodies that mathematics are derived from practice and work for practice. It has reached the organic combination of theory and practice.

Challenging and Interesting. Solving the problems from mathematical modeling contest is a comprehensive examination of college students' mathematical knowledge, computer knowledge, discovery and problem solving ability, information collection ability, writing ability and cooperation ability, etc. It holds great challenging. The contest is highly participatory, enabling students to learn in the activity and create a sense of pleasure and pride, so that the boring sense of mathematics is well restrained.

Problem Representation of Mathematical Modeling. Problem representation is the cognition and understanding of the initial conditions of the problem, the objectives and tasks and their constituent elements. Based on the provided information and their own knowledge and experience, it finds the structure of problems and establishes a problem space. Besides, it is also a process turning external physical stimulation into internal psychological symbols as well as the internalization of problems demonstrated by the subject. The problem characterization process goes through three stages: search and extraction of problem information, understanding and internalization, metaphorical constraint finding. 1) The search and extraction of information is a perceptual process. This perceptual process requires support for verbal knowledge and verbal comprehension, expertise and problem solving experience; 2) understanding and internalization are deep processing of perceived information, which requires basic knowledge, logical thinking and problem-solving skills; 3 ) metaphor constraints find and enrich the need for a wealth of knowledge, association and creativity.

The factors that influence the modeling of mathematical modeling are as follows: 1) The situation of mathematical modeling problem, that is, the way of modeling the mathematical modeling, the environment and its structure, it determines the information that the modeler can perceive and the temporal and spatial structure of these information are the basic information conditions of representation; 2) the knowledge and experience of mathematical modelers, especially the expertise and experience related to the current mathematical modeling problems and the successful experience of solving similar modeling problems. These experiences directly affect the choice of information in the context of modeling problems and the interpretation of perceived information. 3) The quality of thinking and other personality qualities of mathematical modelers, especially the adaptability of thinking, which are of great significance to the problem characterization. 4) Mathematical modelers' emotional state and emotional quality also have a certain impact on the problem.

Mathematical Modeling Strategy based on Cognitive Mechanism. Mathematical modeling strategy is one of the important cognitive components of mathematical modeling, which plays an important role in solving the problem of mathematical modeling. The basic meaning of mathematical 
modeling strategy is to solve the problem of mathematical modeling in the whole process, to think about hypothesis, selection and adoption of solutions and steps of the principles of mathematical modeling problem is a general understanding of the way, principle guiding thought at the macro level. Mathematical modeling strategy has the following basic characteristics: 1) universal adaptability. The mathematical modeling strategy has a higher level of applicability and wider application, and distinguishes it from specific modeling techniques with its overall guiding significance. 2) Direct usability. Mathematical modeling strategy is a bridge of modeling thinking into a modeling operation, which can be used to solve specific modeling problems, and its direct usability is different from abstract modeling ideas. 3) The duality of method. Mathematical modeling strategy is between the specific modeling techniques and abstract modeling ideas, as a method, it is on one hand used as a specific modeling method, on the other hand, it is to find, use, create modeling methods; 4) the optimization of choice. If the mathematical modeling strategy is understood as a series of rules for selecting and combining, these rules should have the basic ability to quickly find the optimal modeling operation, to reduce the number of attempts and failures, to save time to explore, to reflect the choice of wit and combination of art. Mathematical modeling of teaching strategies include the following aspects:

It requires teachers to create conditions for students' re-discovery in the teaching of mathematical modeling, and to provide students with practical opportunities to establish the internal links between the acquired mathematics knowledge and the existing experience. It is necessary to pay attention to the possibility of current learning, but also pay attention to the potential development. The content and method of mathematical modeling teaching activities should meet the students' age characteristics, intellectual development level and psychological characteristics, and are suitable for students' understanding level. It is necessary for students to understand the content and accept the method, but also to enable students' level of knowledge to leap to a new level.

Attention should be paid to the asymptotic teaching in mathematical modeling teaching just like teaching mathematical knowledge. The asymptotic nature of mathematical modeling teaching is mainly manifested as the asymptotic nature of the problem. The problems presented are divided into five levels according to the difficulty and complexity: identification problems, algorithmic problems, application problems, exploration problems and situational problems, in which the identification and algorithmic problems are the basis of mathematical modeling. Mathematical modeling teaching follows the principle of asymptotic. The first stage is the basic application stage. At this stage, the students' modeling knowledge is relatively weak, the modeling consciousness is not strong and there is no mathematical modeling experience, so teachers should carefully select some of the more simple, suitable methods for students to learn the situation of mathematics modeling issues. Teachers and students should establish a mathematical model, but the process should be teacher-led with students as the main body. In the process of modeling, teachers should combine the general meaning, methods and steps of modeling to pay attention to selecting some practical problems that can be solved using only basic mathematical methods as an example, focusing on how to use mathematical knowledge to describe and build models. Students should take the initiative to participate in the mathematical modeling process and pay attention to the cultivation of students' mathematics communication ability, so that students can understand the meaning and method of mathematical modeling, learn the general process and concrete steps of mathematical modeling. The second stage is exploratory construction. In the stage of the model, the teacher should select some typical problems with the characteristics of the situation for students, teachers and students to analyze the actual problem after the mathematical, so that students personally get involved in the modeling process using mathematical knowledge and commonly used modeling methods. Establish and answer the mathematical model according to the actual meaning of the problem and the specific background and test the results of the test. The third stage is the situational modeling stage, and this stage requires students to have a certain degree of mathematical modeling capabilities, to deal with some complex mathematical modeling problems. Generally, only the situation and requirements of problems are given, requiring students to find problems and mine useful information from certain contexts according to the specific situations and basic requirements. Students are required to make assumptions and set some known conditions, propose various model construction methods, get 
the answers and the check, modify and supplement them until the accurate mathematical model is found.

Come active learning and guided learning. Teachers should not only demonstrate mathematical modeling procedures to students, but more importantly, inspire and guide students to find problems, propose problems and solve problems in actual phenomenon. In order to become a good guide in modeling, teachers should pay attention to the following two points: first, they need to understand whether students' existing mathematical development level can make them recall relevant learned knowledge, cognition strategies and problem-solving strategies, etc. Second, they should use well-designed questions to remove thinking obstacles for students or give appropriate directional guidance combined with students' cognition development level. They should carefully design mathematical situations, create an active and harmonious teaching atmosphere to affect students' emotions and then use the emotion' promotion function on cognition to arouse students' interests so that students can take the initiative and become urgent in learning mathematical modeling.

Combine independent inquiry and cooperative exploration. In mathematical modeling teaching, the principle of combing independent inquiry and cooperative exploration should be followed. On one hand, emphasis should be put on developing students' subjective consciousness and students should be encouraged to actively participate in various links of mathematical modeling, think and explore independently so as to propose solutions. On the other hand, attention should be paid to developing students' cooperation awareness and advocating group learning and discussions to give full play to each one's advantages. Simple mathematical modeling can be done independently by students, and complex mathematical modeling can be done using a group of cooperative learning methods.

\section{Summary}

Compared with general problem solving, mathematical problem solving and mathematical application solving, mathematical modeling, as a special way of solving problems, has more vague background and more open structure, and it requires more flexible thinking and better creative results. Researches into the cognitive mechanism of mathematical modeling can help expand the theories of solving mathematical problems, enrich the theories and methods of learning mathematics so as to provide firm learning and cognitive psychological basis for the effective implementation of determining mathematical modeling courses' targets, content systems, compiling textbooks, selecting and applying teaching methods and evaluating teaching and learning. Based on research of mathematical modeling's cognition mechanism, this paper carries out research into the strategies of teaching mathematical modeling, which is helpful for improving the theoretical connotation of mathematical modeling, enriching and deepening mathematics teaching theories and guiding deeper researches into mathematical modeling so as to provide practical guidance for mathematical modeling teaching, greatly promote the publicity and popularity of mathematical modeling course, thus promoting reform of mathematics in basic education.

\section{References}

[1] Larkin,J.H.andSimon,H.A.Why a dlagram is worth ten thousand words. Cognitive Science.2015 (l):85-100.

[2] Metcalfe J.,Wiebe D. Intuition in insight and noninsight problem solving. Memory\& Cognition, 2014, 15:238-246.

[3] Nelson TO. Consciousness and metacognition. American Psychologist, 2016, 51 (2): 102-116.

[4] Armstrong. The influence of individual cognitive style on performance in management edueation.Educational Psyehology, 2017, 20(3):324-338.

[5] H.L.Swanson.Influence of metacognitive knowledge and aptitude on problem solving. Journal of Educational Psychology, 2015, 82(2):306-314. 